Textures and Microstructures, 1988, Vols. 8 \& 9, pp. 483-492

Reprints available directly from the publisher

Photocopying permitted by license only

(C) 1988 Gordon and Breach Science Publishers Inc.

Printed in the United Kingdom

\title{
Statistical Treatment of Measured Orientation Relationships
}

\author{
W. PRANTL, E. WERNER, H. P. STÜWE \\ Erich-Schmid-Institut für Festkörperphysik der Österr. Akademie der \\ Wissenschaften, A-8700 Leoben, Austria
}

(Received May 13, 1987)

Dedicated to the memory of Professor Günter Wassermann

Using the $\chi^{2}$-test a statistical method is described, which allows a clear decision whether a given distribution of orientation relationships between adjacent grains is random or not. The validity of this method even for small numbers of orientation relationships, as obtained from electron microscopical measurements, is shown by generating a small sample of randomly distributed orientation relationships and testing it against the calculated distribution. The method is applied to orientation relationships measured between $\alpha / \alpha-, \alpha / \beta$ - and $\beta / \beta$-neighbouring grains in singleand dual-phase brasses.

KEY WORDS: Orientation relationship, electron diffraction, brass, cubic symmetry, statistcal relevance, $\chi^{2}$-test, random distribution.

\section{INTRODUCTION}

Analysing the flow stress of single and dual phase brasses according to Hall-Petch, it can be seen that-for equal grain sizes-the flow stress of dual phase $\alpha+\beta$-brasses is always higher than that of either single phase $\alpha$ - or $\beta$-brasses. The contributions to the flow stress caused by grain- and phase-boundaries can be separated. It turns out that $\alpha / \beta$-phase boundaries impede the motion of dislocations more effectively than $\alpha / \alpha$ - and $\beta / \beta$-grain boundaries (Werner 
and Stüwe, 1984/85). From bicrystal experiments (Hingwe and Subramanian, 1975) it can be deduced that the orientation relationship between neighbouring crystals influences the efficiency of grain and phase boundaries as dislocation obstacles. In polycrystals, however, many different orientation relationships between neighbouring grains are present. In order to investigate the influence of those relationships on the behavior of grain- and phase-boundaries it is necessary to know the orientation relationships between a large number of neighbouring grains. Since this information can only be obtained by electron microscopy techniques the experimental effort is great. In this paper a statistical method is presented, which allows to draw conclusions from a limited number of measured orientation relationships.

\section{DETERMINATION OF ORIENTATION RELATIONSHIPS}

The materials used for our investigations are single and dual phase brasses with varying volume fractions of the $\alpha$ - and $\beta$-phases. Cold rolled and rotary swaged rods of our alloys are recrystallized. The rods are cut to discs, then ground to $0.1 \mathrm{~mm}$ thickness and electrolytically polished (double jet polishing:

$$
\mathrm{HNO}_{3}: \mathrm{CH}_{3} \mathrm{OH}=1: 3, \quad T=-60^{\circ} \mathrm{C}, \quad U=12 \mathrm{~V} ;
$$

final polishing: $\mathrm{H}_{3} \mathrm{PO}_{4}: \mathrm{H}_{2} \mathrm{O}=3: 5, T=+10^{\circ} \mathrm{C}, U=2 \mathrm{~V}$ ). The measurements are performed using a double-tilt goniometer stage, where the specimen can be tilted about two mutually perpendicular axes $I$ and $I I$. Axis $I$ is mounted horizontally and is fixed with respect to a microscope coordinate system, while axis $I I$ can be tilted about axis $I$ and is fixed with respect to a specimen coordinate system. In order to determine the orientation relationship between two adjacent grains three diffraction spot patterns with arbitrary zone axes are taken under Laue conditions from each grain and the respective goniometer readings, i.e. the orientations of tilt axes $I$ and II, are noted. By means of a computer program (Prantl, 1984) the indices of the foil normal $\overrightarrow{F N}$ and of the tilt axis $I I$ are calculated for each crystal. Since these two directions are fixed with respect to the specimen they define a specimen coordinate system.

The indices of these two directions are expressed in the coordinates of both grains, namely $\overline{F N}(A), \overline{I I}(A)$ for crystal $A$ and $\overline{F N}$ 
$(B), \overrightarrow{I I}(B)$ for crystal $B$. Since these two sets of indices describe the very same directions in real space we can describe the rotation of grain $A$ into grain $B$ by the matrix

$$
R=M_{B}^{-1} M_{A}
$$

where $M_{A}$ and $M_{B}$ are the matrices which perform the rotation of grain $A$ and grain $B$ into the system of reference, respectively. This matrix of rotation $R$ therefore characterizes the orientation relationship between grains $A$ and $B$. Both, the $\alpha$ - and $\beta$-phases of our brasses belong to the cubic crystal system. According to the cubic symmetry we obtain 24 matrices of rotation because there are 24 different ways to define a right-handed coordinate system in a cube. These matrices represent 24 equivalent descriptions of a single physical orientation relationship.

In order to be able to compare orientation relationships of different pairs of grains we choose a different method for the description of orientation relationships. For each matrix of rotation we find a pair consisting of an axis of rotation $\vec{P}$ and an angle of rotation $\omega$ describing the same rotation. $\vec{P}$ is the eigenvector of the eigenvalue +1 and is evaluated from the trace of $R$, namely

$$
\omega=\arccos \left(\left(r_{11}+r_{22}+r_{33}-1\right) / 2\right) \text {. }
$$

Because of the cubic symmetry we find 24 sets of axes and angles for each orientation relationship. The smallest of the 24 angles of rotation so obtained is usually called the angle of disorientation, the corresponding axis the axis of disorientation (Mackenzie, 1964). We shall consider this particular set to describe our results.

\section{RESULTS}

Figure 1 shows the distribution of angles of disorientation as obtained from experiment. Figure 2 shows the distribution of directions of the axes of disorientation as obtained from experiment. The results for $\alpha / \alpha-, \alpha / \beta$ - and $\beta / \beta$-boundaries are plotted separately. If we want to know whether these distributions should be considered as "random" we must compare them to the distribution functions for random orientation. These have been calculated by Handscomb (1958) and Mackenzie (1958) and are shown in Figure 3 and 4 . A comparison by simple inspection appears 


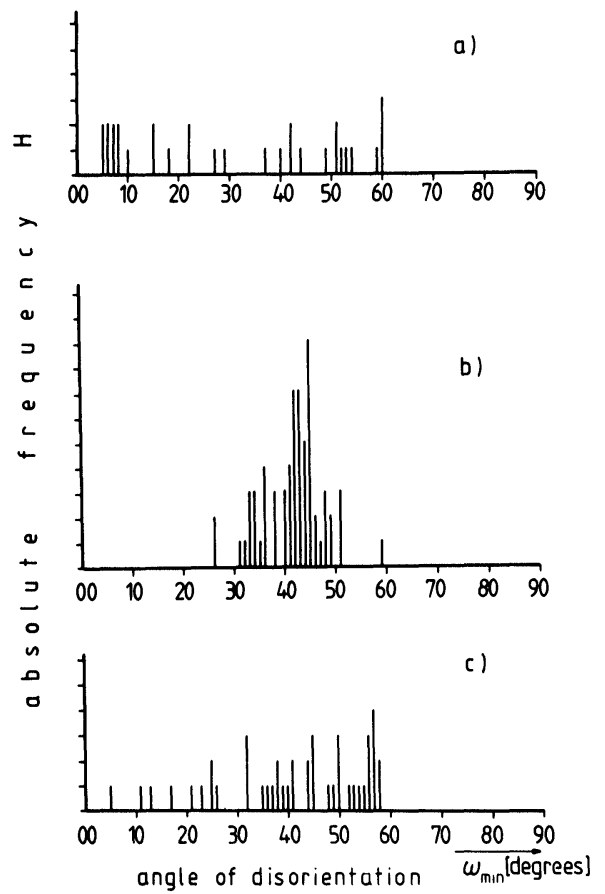

Figure 1a-1c Frequency distribution of the measured angles of disorientation $\omega_{\min }$ for $\alpha / \alpha-, \alpha / \beta$ - and $\beta / \beta$-neighbours.

hopeless. The number of our experiments is too small to permit even an approximation of a smooth distribution function. There is, however, a more powerful statistical tool to permit such a comparison: it is the $\chi^{2}$-test described in short in the appendix. It shall be applied both to our experiments and to equally small samples produced by a random generator.

\section{GENERATION OF RANDOM ORIENTATION RELATIONSHIPS AND COMPARISON TO EXPERIMENT}

The basic idea is to fix one cube and then throw the second one into arbitrary orientations of equal probability in space. Each of these orientations can be described with respect to the first cube by an 

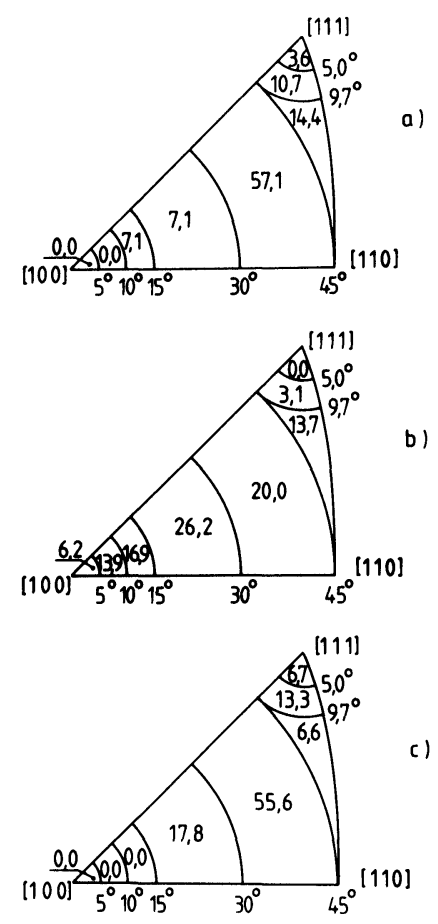

Figure 2a-2c Distribution of the measured axes of disorientation in the unit triangle for $\alpha / \alpha-, \alpha / \beta$ - and $\beta / \beta$-neighbours.

axis of rotation $P$ and an angle of rotation $\omega$. The orientations can be randomly distributed only if the related axes and angles of rotation are randomly distributed, too. So, creating randomly distributed orientation relationships means distributing axes over all directions in space with equal probability and for each axis creating an angle of rotation $\omega$ uniformly distributed between 0 and 360 degrees.

In order to meet each surface element on a sphere of unit radius with equal probability the size of the surface elements must be independent of their position on the sphere. This condition is met if the component $w$ of the axis is a uniformly distributed random number between -1 and +1 (see Figure 5). By means of the angle $\varphi$, created as uniformly distributed random number between 0 and 


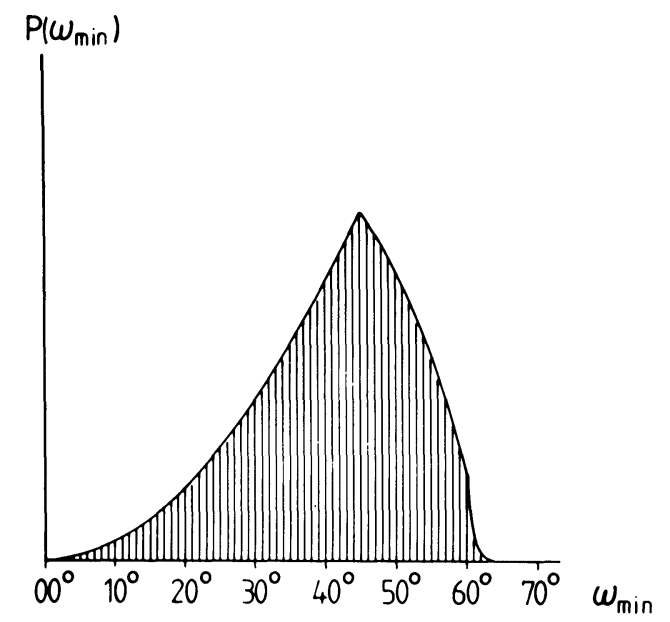

Figure 3 Frequency distribution of the angles of disorientation $\omega_{\min }$ for randomly distributed orientation relationships. Solid curve: as calculated by Mackenzie. Vertical lines: as obtained for a sample of one million orientation relationships generated by a computer.

360 , one obtains the components $u$ and $v$, namely

$$
u=\left(1-w^{2}\right) \frac{1}{2} \cdot \cos \varphi, \quad v=\left(1-w^{2}\right) \frac{1}{2} \cdot \sin \varphi .
$$

The angle of rotation $\omega$ belonging to this axis $[u v w]$ is generated as uniformly distributed random number between 0 and 360 .

An equivalent method of creating randomly distributed orienta-

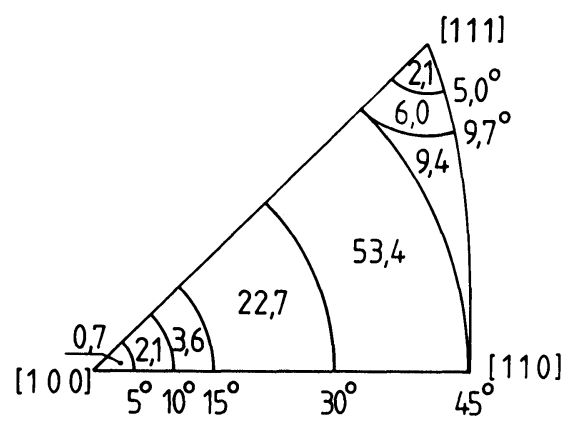

Figure 4 Distribution of the axes of disorientation in the unit triangle for randomly distributed orientation relationships (Mackenzie, 1964). 


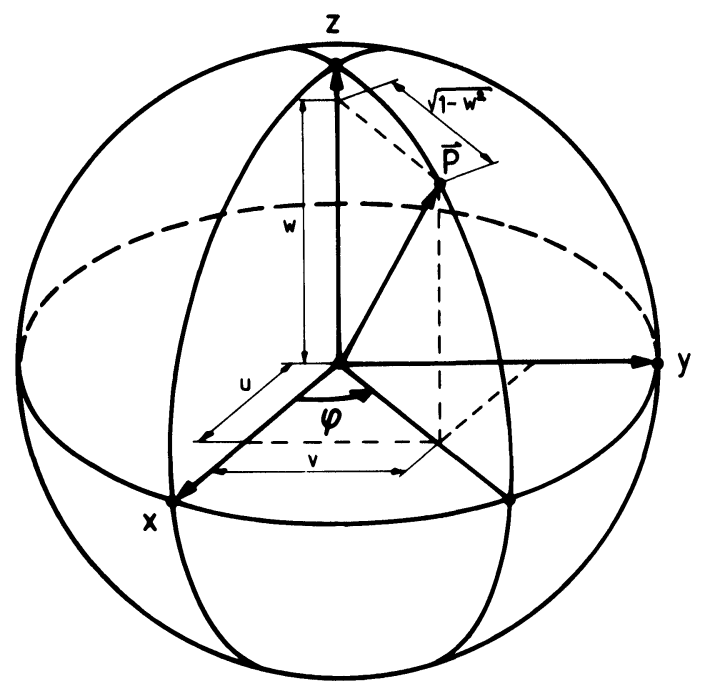

Figure 5 Generation of randomly distributed axes in space.

tion relationships is to generate three Euler-angles. In this case the angles $\varphi_{1}$ and $\varphi_{2}$, which represent rotations about the old and the new $z$-axis, respectively, are created uniformly distributed between 0 and 360 degrees. The angle $\phi$ between the old $z$-axis and the new one, however, has to be weighted by an arccos-function between 0 and 180 degrees.

Either from the generated axis/angle-set or from the generated Euler-angles the matrix of rotation can be evaluated, which describes the orientation relationship. From the generated matrix of rotation the angle and the axis of disorientation are calculated as previously described in our paper. For the sake of completeness we would like to mention that the axes of disorientation are not uniformly distributed on the sphere. To be sure that this method really creates randomly distributed orientation relationships a sample of one million orientation relationships was generated. Figure 3 shows the resulting distribution compared to Mackenzie's distribution. The excellent agreement is obvious. Then, small samples of about 40 random orientations were generated by the same program. Here it is not obvious that this small sample agrees with Mackenzie's distribution. Applying the $\chi^{2}$-test, however, shows 
that the sample can be safely considered as randomly distributed. Applying the same test to our results from Figures 1 and 2 we obtain the result that none of the distributions shown there can be considered as random.

\section{Acknowledgement}

The authors would like to thank $H$. Schütz for the careful preparation of the thin foils and H. G. Kopetzky for valuable discussions concerning the $\chi^{2}$-test.

\section{References}

1. Handscomb, D. C., Canad. J. Math. 10, 85-88 (1958).

2. Hingwe, A. K. and Subramanian, K. N., J. Mater. Sci. 10, 183-188 (1975).

3. Kreyszig, E., Statistische Methoden und ihre Anwendung, 4. Auflage, Vandenhoeck u. Ruprecht, Göttingen (1974).

4. Mackenzie, J. K., Biometrika 45, 229-240 (1958).

5. Mackenzie, J. K., Acta Met. 12, 223-225 (1964).

6. Prantl, W., J. Appl. Cryst. 17, 39-42 (1984).

7. Werner, E. and Stüwe, H. P., Mater. Sci. Eng. 68, 175-182 (1984/85).

\section{APPENDIX: THE $\chi^{2}$-TEST}

To explain the principles of the test we consider the distribution of the angle of disorientation for the $\alpha / \beta$-orientation relationships (see Figure $1 \mathrm{~b}$ ): The $\omega_{\min }$-axis is subdivided into $K$ intervals $I_{j}$ so, that each interval contains at least four values of the sample. Their number $b_{j}$ is noted for all intervals.

Using Mackenzie's distribution function (Mackenzie 1958) we compute the probabilities $p_{j}$ to find a value in those intervals and from these $p_{j}$ the number $e_{j}=n p_{j}$ of the values one expects to lie within $I_{j}(n$ is the sample size = number of measured orientation relationships). Then we calculate the deviation $\chi_{0}^{2}$ between the sample and the theoretical distribution:

$$
\chi_{0}^{2}=\sum_{j=1}^{K} A_{j}=\sum_{j=1}^{K} \frac{\left(b_{j}-e_{j}\right)^{2}}{e_{j}}
$$

For large $n$ the distribution function of the random variable $\chi_{0}^{2}$ approaches the $\chi^{2}$-distribution function with $K-1$ degrees of freedom (Kreyszig, 1974). The numeric values in Table 1 refer to the test performed with the measured $\alpha / \beta$-data. 
Table 1 The $\chi^{2}$-test for the distribution of $\omega_{\min }$ for $\alpha / \beta$-orientation relationships $(K=11, n=65)$

\begin{tabular}{lllrl}
\hline Intervals for & & & & \\
$\omega_{\min }$ [degrees] & $b_{j}$ & \multicolumn{1}{l}{$p_{j}$} & \multicolumn{1}{c}{$e_{j}$} & \multicolumn{1}{c}{$A_{j}$} \\
\hline $0-32.5$ & 4 & 0.229 & 14.9 & 7.95 \\
$32.5-34.5$ & 6 & 0.044 & 2.9 & 3.38 \\
$34.5-37.5$ & 5 & 0.076 & 5.0 & 0 \\
$37.5-40.5$ & 6 & 0.089 & 5.8 & 0.01 \\
$40.5-41.5$ & 4 & 0.033 & 2.1 & 1.64 \\
$41.5-42.5$ & 7 & 0.034 & 2.2 & 10.20 \\
$42.5-43.5$ & 7 & 0.036 & 2.3 & 9.36 \\
$43.5-44.5$ & 5 & 0.037 & 2.4 & 2.72 \\
$44.5-45.5$ & 9 & 0.039 & 2.5 & 16.66 \\
$45.5-48.5$ & 6 & 0.109 & 7.1 & 0.17 \\
$48.5-59.5$ & 6 & 0.260 & 16.9 & 7.06 \\
\hline$\chi_{0}^{2}=59.33$. & & & &
\end{tabular}

We then choose the significance level $\alpha$ of the test. $\alpha$ has the meaning of a probability and is commonly chosen to be 0.05 . The solution $c$ of the probability-equation:

$$
P\left(\chi^{2} \leqslant c\right)=1-\alpha
$$

is found using the $\chi^{2}$-distribution with $K-1$ degrees of freedom. The procedure is shown in Figure A1, where the density function of

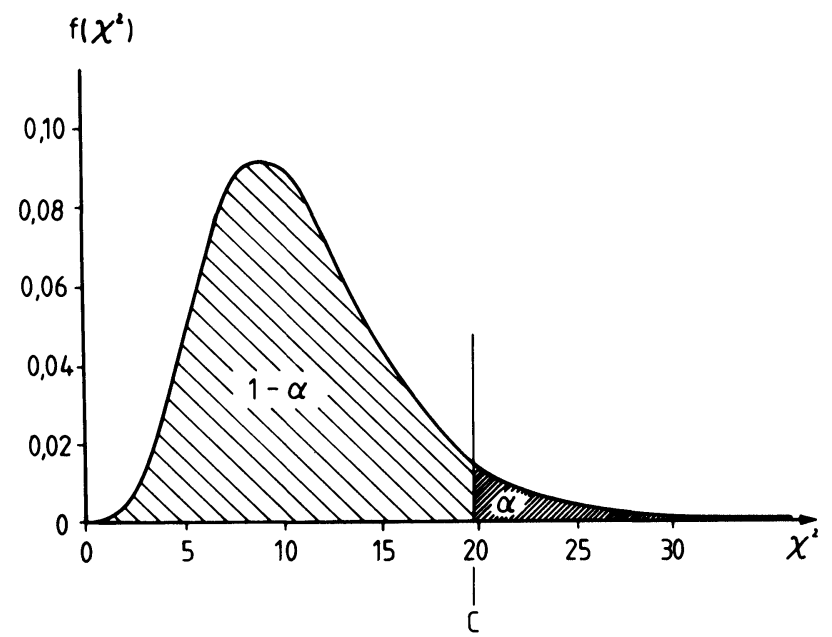

Figure A1 Density function of the $\chi^{2}$-distribution and the meaning of the significance level $\alpha$. 
the $\chi^{2}$-distribution is plotted against the random variable $\chi^{2}$. Since areas underneath this curve have the meaning of probabilities, we see that $\alpha$ is the probability that the random variable $\chi^{2}$ takes values greater than $c$. If $\chi_{0}^{2}$ computed from the sample is situated to the left of $c$, i.e. $\chi_{0}^{2} \leqslant c$, the hypothesis that the sample obeys the theoretical distribution will be accepted. In the case of $\chi_{0}^{2}>c$ this hypothesis will be rejected and for $\chi_{0}^{2} \ll c$ and $\chi_{0}^{2} \gg c$ it will be "clearly" accepted and "clearly" rejected, respectively.

In our example the random variable $\chi_{0}^{2}$ is $\chi^{2}$-distributed with 10 degrees of freedom. For $\alpha=0.05 c$ turns out to be 18.31 (Kreyszig, 1974). Since $\chi_{0}^{2}=59.33 \gg c$ the hypothesis that our $\alpha / \beta$-orientation relationships obey Mackenzie's random distribution must be "clearly" rejected.

The small value for $\alpha$ can be explained as follows: For a large $\alpha$ ( $\Rightarrow c$ very small!) the hypothesis has to be rejected although it is true, because the sample yields a value for $\chi_{0}^{2}$ which does not satisfy the inequality $\chi_{0}^{2} \leqslant c$. There are two ways this wrong decision can be made less probable: One can increase the sample size. This is not possible in many cases because of the increased experimental effort. The second possibility is to choose small values for $\alpha$. By means of plots like Figure A1 one can then decide from the values of $\chi_{0}^{2}$ and $c$ if the hypothesis will be "clearly" accepted, accepted, rejected or "clearly" rejected. 